\title{
Estimation of subject specific ICP dynamic models using prospective clinical data
}

\author{
W. Wakeland ${ }^{1,2}$, J. Fusion ${ }^{1} \&$ B. Goldstein ${ }^{3}$ \\ ${ }^{I}$ Systems Science PhD Program, Portland State University, Portland, \\ Oregon, U.S.A. \\ ${ }^{2}$ Biomedical Signal Processing Laboratory, \\ Department of Electrical and Computer Engineering, \\ Portland State University, Portland, Oregon, U.S.A. \\ ${ }^{3}$ Complex Systems Laboratory, Doernbecher Children's Hospital, \\ Division of Pediatric Critical Care, Oregon Health \& Science University, \\ Portland, Oregon, U.S.A.
}

\begin{abstract}
We used a prospective clinical trial to generate physiologic data to create subject-specific in silico (computer simulation) models of intracranial pressure dynamics in children with severe traumatic brain injury. The trial included a physiologic challenge protocol with changes in head-of-bed elevation and minute ventilation, applied over multiple iterations to three subjects. Physiologic signals (electrocardiogram, respiration, arterial blood pressure, intracranial pressure [ICP], oxygen saturation) were recorded continuously, along with clinical annotations indicating the precise timing of physiologic challenges. Several parameters within the model of ICP dynamics were estimated for each subject based on the ICP response to the challenges. Estimation was done using a standard optimization algorithm to minimize the difference between the ICP trajectory predicted by the model and the actual ICP data. The ICP trajectory predicted by the model was similar to the actual ICP data in all cases, and the mean absolute error varied between $0.5-2.8 \mathrm{mmHg}($ mean $=1.4 \mathrm{mmHg})$. These results demonstrate the potential for using clinically annotated prospective data to create subject-specific computer simulation models. Future research will focus on improvements in the logic for cerebral autoregulatory mechanisms and physiologic adaptation.

Keywords: intracranial pressure, simulation model, clinical data, optimization.
\end{abstract}




\section{Introduction}

Our goal is to develop tools that can be used improve the care of children with severe traumatic brain injury (TBI). Section 2 provides background material regarding the importance of the problem - the need to improve our understanding of intracranial pressure (ICP) dynamics during TBI. A brief review of prior research is also provided. Our method, described in Section 3, is to create subject-specific in silico ICP dynamic models using clinically annotated data of each subject's response to a physiologic challenge protocol. The results of the study are documented in Section 4 and are presented in both tabular and graphical formats. A discussion of the results is provided in Section 5.

\section{Background}

TBI is the leading cause of death and disability in children under 18 years old, causing more than $50 \%$ of all childhood deaths. Each year, more than 150,000 pediatric brain injuries result in about 7,000 deaths and 29,000 children with new, permanent disabilities. The death rate for severe TBI (defined as a Glasgow Coma Scale score $\leq 8$ ) remains between $30 \%-45 \%$ at major children's hospitals [1] [2]. A recently published evidence-based medicine review reports that elevated pressure in the brain (ICP) is a main determinant of outcome following TBI and is strongly correlated with both death and disability [3].

Despite the availability of many treatment options for reducing elevated ICP (defined as $\geq 20 \mathrm{mmHg}$ ), poor outcomes still occur, often due to elevated ICP that is unresponsive to therapy. Treatment options for severe TBI include draining cerebral spinal fluid (CSF) via a ventriculostomy catheter, raising the head-of-bed (HOB) elevation to $30^{\circ}$ to promote jugular venous drainage, and inducing mild hyperventilation [3]. The underlying pathophysiologic mechanisms governing ICP regulation and the mechanisms by which treatment affects ICP remain poorly understood [4].

We suggest that new approaches are needed to help improve diagnosis, treatment, and outcome following severe TBI. We hypothesize that an in silico model of ICP dynamics to test various therapeutic options for elevated ICP in a virtual (computer simulated) patient before treating the actual patient will improve care of acute elevations in ICP and may also improve long-term outcome following severe TBI.

\subsection{Prior work by other researchers}

Over the past 30 years, a variety of computer models for calculating ICP dynamics have been published in the biomedical engineering literature (c.f. [5-8]). These models use differential equations to calculate the pressure at different points within the system, taking into account the blood vessel and anatomic compartment volumes and compliances. Although useful mathematical results and insights have been gained from these models, their impact on clinical practice has been modest. Reasons for this limited impact may include the complexity of the models and the limited range of bedside in vivo clinical 
scenarios represented. Researchers have attempted to address these limitations by simplifying their models (c.f. [9]) and adding needed functionality.

\subsection{Prior work by the investigators}

In our subject-specific in silico model, fluid volumes and flows are explicit within the model and we incorporate specific physiologic parameters and mechanistic logic. The resultant model [10] is similar in many ways to previously reported dynamic ICP models in that it considers the cranial vault to contain the brain parenchyma plus several fluid "compartments" that together are constrained not to exceed the total cranial volume. The fluid compartments include the arterial blood volume, capillary blood volume, venous blood volume, CSF volume, the brain volume, and "other" volume that may or may not be present in a particular patient (e.g. epidural hematoma, subdural hematoma, intraparenchymal hemorrhage, etc.). In our model, brain volume can be either constant or variable, based on the presence or absence and degree of cerebral edema.

As with previously published models, we model cerebral autoregulation (a key physiologic mechanism) as a feedback loop that causes the cerebral vasculature to dilate or constrict, taking into account control limits that are nonlinear and asymmetric. Our control logic acts only on the flow of blood from the arterial compartment to the capillary bed. The control logic is proportional and has enough "gain" that it can easily maintain the required flow under normal conditions. However, if the venous or arterial volumes are severely reduced, as is often the case with severe TBI, the associated nonlinear increases in resistance can "overwhelm" the model's simplified control logic in an unrealistic fashion.

The main difference between our in silico ICP dynamic model and previously reported models is that the state variables are modeled as volumes rather than pressures, and we clearly identify fluid flows into and out of each compartment. This approach is more intuitive and may more accurately represent in vivo pathophysiologic processes. Blood pressures are computed from the volumes of the blood compartments and their associated compliances. ICP (the CSF pressure within the cranial vault) is computed using the total intracranial volume and the pressure volume index [5].

Our model incorporates logic associated with severe TBI pathophysiology, including the "other" volume mentioned above, ongoing intracranial bleeding, and focal or generalized cerebral edema. The model also incorporates common therapeutic interventions such as elevation of the HOB and changing the minute ventilation to induce mild hyperventilation (decreasing $\mathrm{PaCO}_{2}$ to $33-35 \mathrm{mmHg}$ ). We have previously shown that the behaviour of our model was qualitatively correct [11], and we were able to manually calibrate the model so that it replicated clinical data from two prior subject-specific cases [12].

\section{Methods}

A physiologic challenge protocol was applied over multiple iterations to three subjects with severe traumatic brain injury. Parameters were then estimated to 
configure a computer model of ICP dynamics to the specific characteristics of each subject.

\subsection{Physiologic challenge protocol and data collection}

This study was approved by the Institutional Review Board at Oregon Health \& Science University. Following informed consent, physiologic challenges were randomly administered over a 2-3 hour period. These included:

1) Altering the head of the bed (HOB) elevation in random order between $0^{\circ}$ and $40^{\circ}$, in $10^{\circ}$ increments or decrements at 10 -minute intervals.

2) Increasing minute ventilation (hyperventilation) or decreasing minute ventilation (hypoventilation), in random order, while keeping the tidal volume fixed to achieve an end-tidal $\mathrm{CO}_{2}\left(\mathrm{ETCO}_{2}\right)$ of $[-3$ to -4$] \mathrm{mmHg}$ and $[+3$ to +4$]$ $\mathrm{mmHg}$ from baseline values. At each target $\mathrm{ETCO}_{2}, \mathrm{PaCO}_{2}$ was checked via arterial blood gas to ensure that the ETCO2 reflected accurate changes in actual $\mathrm{PaCO}_{2}$. If not, then minute ventilation was further adjusted, and $\mathrm{ETCO}_{2}$ and $\mathrm{PaCO}_{2}$ were rechecked at each target level of hyper- and hypoventilation was maintained for 15 minutes.

Throughout the challenge protocol, physiologic signals (ICP, blood pressure, electrocardiogram, airway pressure, etc.) were continuously recorded, along with clinical annotations to indicate the precise timing of the physiologic challenges and therapeutic interventions.

\subsection{Model estimation method}

The changes specified by our physiologic challenge protocol served as primary inputs to the ICP dynamic model. These changes to HOB and minute ventilation entered the model as step functions at the times specified in the clinical annotations to the data. There were no other time-based inputs to the model; all other parameters were either initial values or constants. Although the protocol sessions provided multiple physiological signals for each subject, the only measurement used by the model was the ICP signal used to compute error when tuning the model to a subject-specific session.

The ICP signal was captured at $125 \mathrm{~Hz}$. Since the model sought to capture only the time behaviour of mean ICP, the high-frequency components of the signal were ignored. Therefore, the data was decimated and a lowpass filter was applied, yielding smoothed data at a sample rate of $5 \mathrm{~Hz}$, which removed most of the pulsatile component. This data was still noticeably more complex than the output of our model, but our research showed that further simplification threatened to remove important features of the ICP data.

The in silico model of ICP dynamics is patterned after models previously reported in the literature and enhanced through incorporation of subject-specific data from the physiologic challenge protocol. The model was first implemented using the STELLA ${ }^{\circledR}$ modeling software, due to its ease of use for initial conceptualization and experimentation. This platform proved limited in terms of data handling, integration, and incorporation of other algorithms. Consequently, 
the model was ported to MATLAB Simulink ${ }^{\circledR}$. This simulation environment has proven to be more technically robust and easily handles large data sets. Figure 1 shows a block diagram of the model.

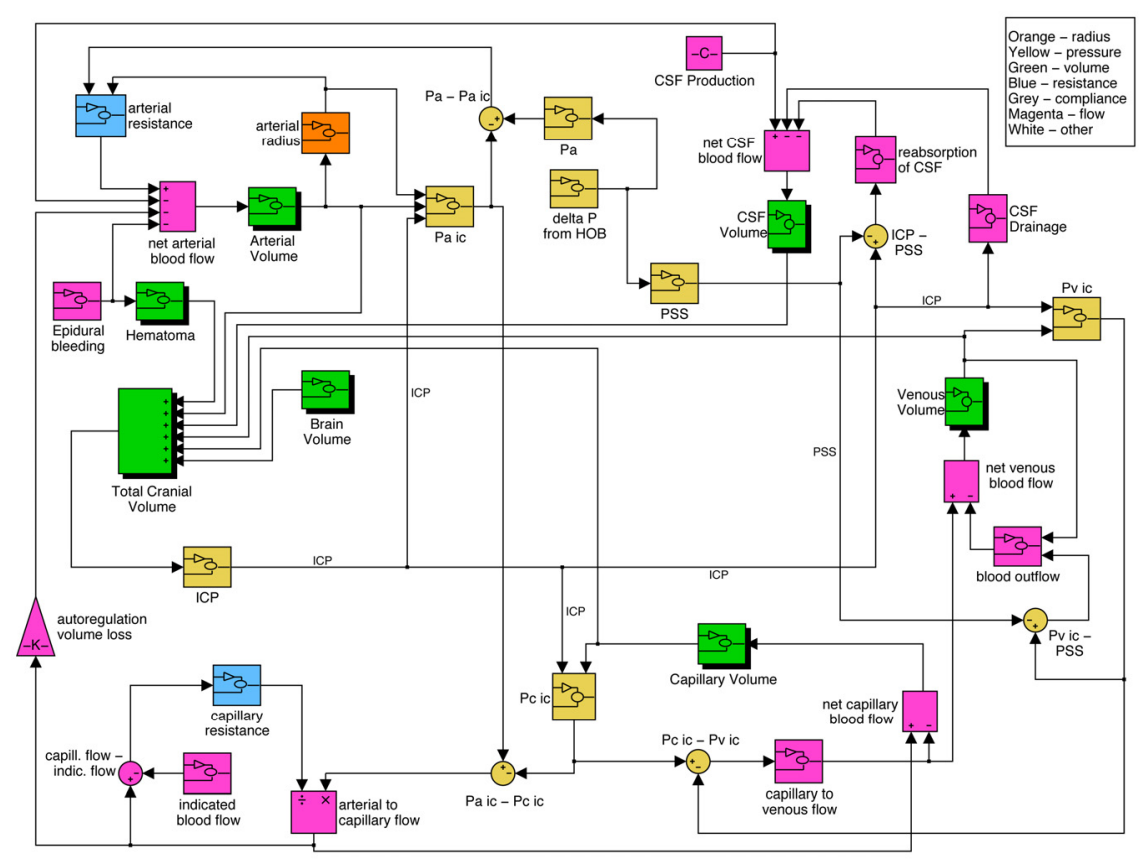

Figure 1: ICP dynamic model, as implemented in Simulink. The primary state variables are the six volumes (arterial, brain, capillary, CSF, hematoma, and venous), represented by the green shadowed "flowchart" icons. Each of the flowchart icons in this figure represents a masked subsystem containing additional model logic.

The state variables in the model are the volumes of the various fluid compartments. Changes in these volumes cause changes in pressure. Pressure changes affect the flows, which in turn impact the volumes. These feedback loops dominate the behavior of the model, and the most influential of them is the ICP loop itself. ICP is a function of the sum of the six volumes in the model. ICP also directly influences four of those volumes: arterial blood, capillary blood, CSF, and venous blood. The other two volumes, representing the brain parenchyma and hematoma, influence ICP but are not influenced by ICP in our model. The inputs from the physiologic challenge protocol are each simulated in the model by specific blocks for that purpose.

The underlying physiology of these systems is very complex and not completely understood, so our model is necessarily a simplification of in vivo processes. In our model, changing the angle, $\theta$, of the HOB changes arterial pressure and ICP by an amount that is proportional to $\sin (\Delta \theta)$. Relative to HOB 
changes, the effects of changing minute ventilation are much more complex. Minute ventilation is the product of the respiratory frequency (or respiratory rate, $\mathrm{RR}$ ) and tidal volume (the volume of inspired air with each breath). As tidal volume was held constant in all the physiologic challenge protocols, we have used RR to represent any changes in minute ventilation. Changes in RR directly affect $\mathrm{PaCO}_{2}$, which is a vital part of the cerebral autoregulation mechanismanother complex feedback loop in our model. An increase in RR causes a decrease in the indicated blood flow and a subsequent increase in capillary resistance, through the mechanism of the $\mathrm{PaCO}_{2}$ effect on smooth muscle tone. The increase in capillary resistance decreases the arterial-to-capillary flow to match the indicated flow, and also affects arterial blood volume. This loop is problematic, as will be discussed later.

Besides the state variables (volumes) and inputs (physiologic protocol challenge data), the other dynamic quantities in the model are the parameters used for fitting. These physiologic parameters were estimated for each subject based on the measured ICP response. The estimation was done by varying the parameters with the objective of minimizing the squared error between the ICP trajectory predicted by the model and the actual ICP data. This optimization was performed using single parameters during sensitivity testing and model exploration. After the effects of the different parameters were better understood, several of them were selected for use with a nonlinear optimization algorithm, MATLAB's lsqcurvefit. This algorithm was allowed to run either until error reduction ceased or until a certain number of iterations had been completed. The algorithm required many simulations of the model to be run, each with slightly different parameter values. Since each run can take several minutes to complete, the entire nonlinear optimization process can require up to several hours.

The process of fitting the model to the data also involved making adjustments to the parameters of the differential equation solver. The model is sensitive to the sample time used by the solver, such that under certain conditions and certain parameters the solver caused one of the state variables to exceed its bounds. Errors sometimes occurred because the volumes in the model are very small relative to the flow over a given length of time. This can cause a volume to temporarily become zero or negative during the calculation update interval, leading to mathematical errors in the solver and erratic behavior in the model. In order to avoid these errors, the sample time of the solver was maintained as low as $1 / 18,000 \mathrm{~min}$. However, to decrease the computational requirements, the sample time was maximized when possible. In some simulations, the much larger sample time of $1 / 900 \mathrm{~min}$. was found to be acceptable.

\section{Results}

We found that the ICP trajectory predicted by the subject-specific in silico model was qualitatively very similar to the in vivo ICP data in all cases. The predicted trajectories matched the ICP data best following HOB elevation challenges, frequently achieving mean absolute errors of $<1.0 \mathrm{mmHg}$. The simulations of RR 
challenges did not perform quite as well, but were still able to capture major features of ICP behavior.

Table 1 compiles the results of several of the model's fitting sessions. In each case, the nonlinear optimization algorithm was allowed to vary 6-8 parameters. The parameters were chosen from the following group based on sensitivity testing and their applicability to the particular challenges of the session: autoregulation volume loss, basal cranial volume, CSF drainage rate, hematoma increase rate, $\Delta$ pressure time constant (a smoothing parameter associated with $\mathrm{HOB}$ elevation change), $\mathrm{ETCO}_{2}$ time constant (a smoothing parameter associated with RR changes), smooth muscle gain (a multiplicative factor related to the impact of smooth muscle tension), and systemic venous pressure. The fitting process was run for several iterations, or until the change in the sum of the squared errors fell below a certain tolerance. The objective function was to minimize the sum of squared errors, ignoring some outliers (for example, at the start of a simulation run or where the actual ICP data showed likely measurement discrepancies). Figures 2 to 5 show example results graphically.

Table 1: $\quad$ Results of model fitting to 8 subject sessions.

\begin{tabular}{|l|c|c|c|l|}
\hline $\begin{array}{l}\text { Subject \& } \\
\text { Session }\end{array}$ & $\begin{array}{l}\text { Session } \\
\text { Length } \\
\text { (minutes) }\end{array}$ & $\begin{array}{l}\text { Mean } \\
\text { Absolute Error } \\
(\mathrm{mmHg})\end{array}$ & $\begin{array}{l}\text { Approximate } \\
\text { Fitting Time } \\
\text { (minutes) }\end{array}$ & $\begin{array}{l}\text { Number and } \\
\text { Types of } \\
\text { Challenges }\end{array}$ \\
\hline P1, S1.5 & 12 & 0.923 & 5 & 1 HOB \\
\hline P1, S3 & 18 & 0.906 & 8 & 1 HOB \\
\hline P1, S4 & 46 & 1.689 & 20 & 1 HOB, 3 RR \\
\hline P2, S1 & 58 & 0.570 & 30 & 4 HOB \\
\hline P2, S4 & 67 & 0.486 & 120 & 2 RR \\
\hline P2, S7 & 110 & 2.337 & 180 & 5 HOB, 4 RR \\
\hline P2, S7a & 55 & 1.624 & 90 & 5 HOB \\
\hline P2, S7b & 55 & 2.810 & 90 & 4 RR \\
\hline & & Mean $=1.418$ & & \\
\hline
\end{tabular}

\section{Discussion}

Although the in silico model was able to accurately predict ICP responses to HOB changes, the model was less accurate when predicting responses to RR changes. Specifically, the model was unable to predict systemic adaptation, as when sequential events diverged from, and then returned to, a particular value. For instance, in P2S7 (see Figure 5), when HOB moved from $30^{\circ}$ to $0^{\circ}$ and back to $30^{\circ}$, the ending in vivo ICP was lower than its original value. However, in the model, ICP returned to its original value. Another inadequacy of the model occurred when a series of events were temporally clustered. These events did not have the same effect on the actual ICP as when they were temporally dispersed, and the model was not able to capture that interaction. Finally, the model was not able to display the range of amplitudes associated with RR physiologic protocol 
challenges. We believe this is due at least partially to deficiencies in the autoregulation logic in the model, such that changes in smooth muscle tone affect the resistance of arterial-to-capillary blood flow but do not explicitly affect arterial volume.

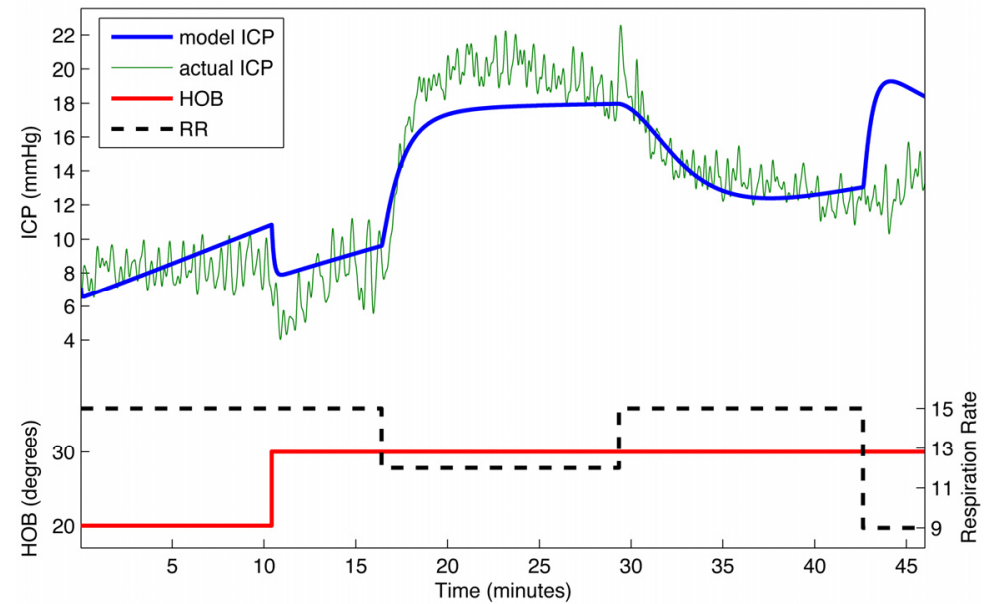

Figure 2: $\quad$ Patient 1, Session 4. A series of changes to HOB elevation and RR.

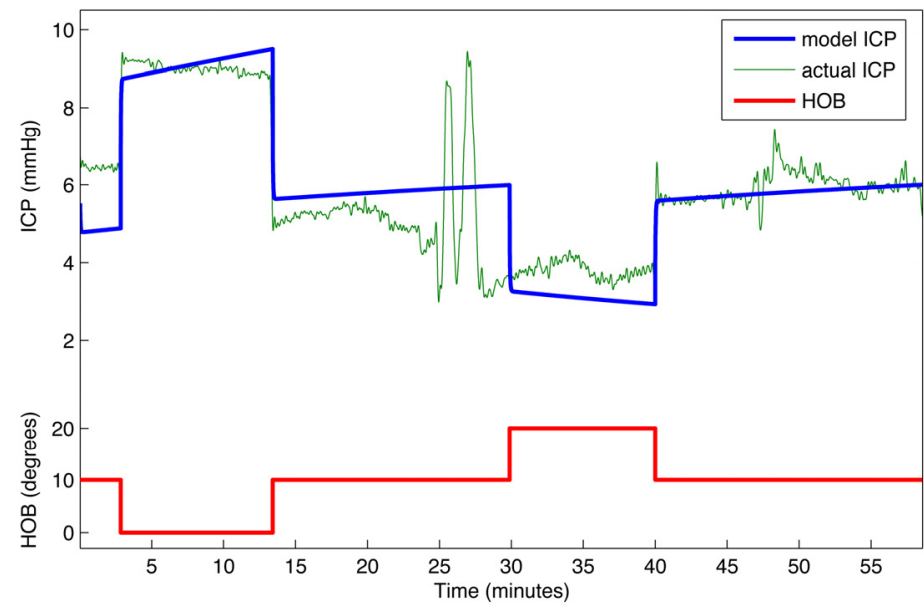

Figure 3: Patient 2, Session 1. A series of changes to HOB elevation.

In summary, our findings indicate the need to add systemic adaptation logic to the model and revise the cerebral autoregulation logic. Data from additional subjects will help to further our understanding of cerebral pathophysiology and to generalize the in silico model to fit multiple clinical conditions. Additional data will also aid in the selection of optimization parameters and processes. 


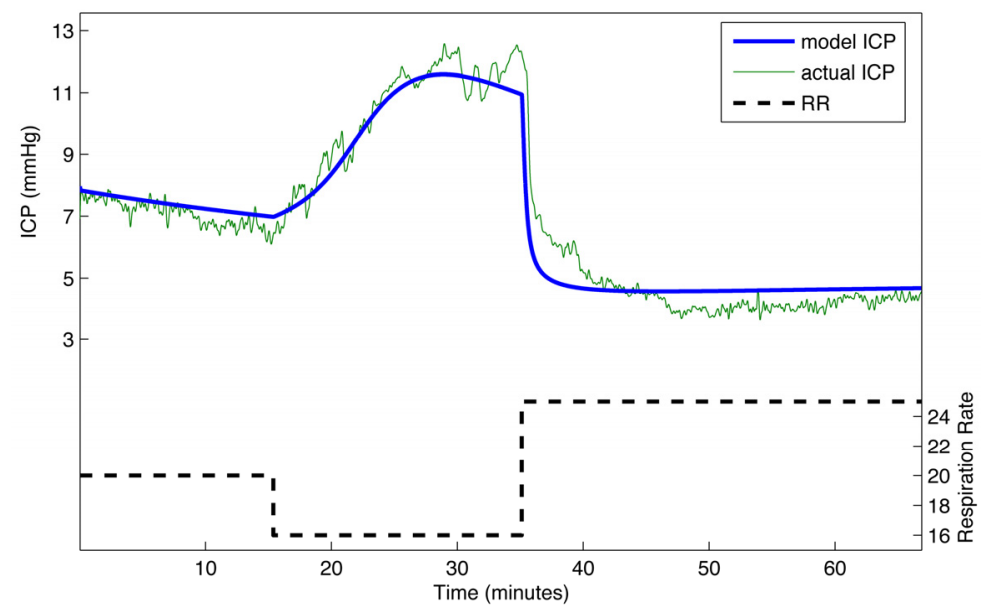

Figure 4: Patient 2, Session 4. A series of changes to RR.

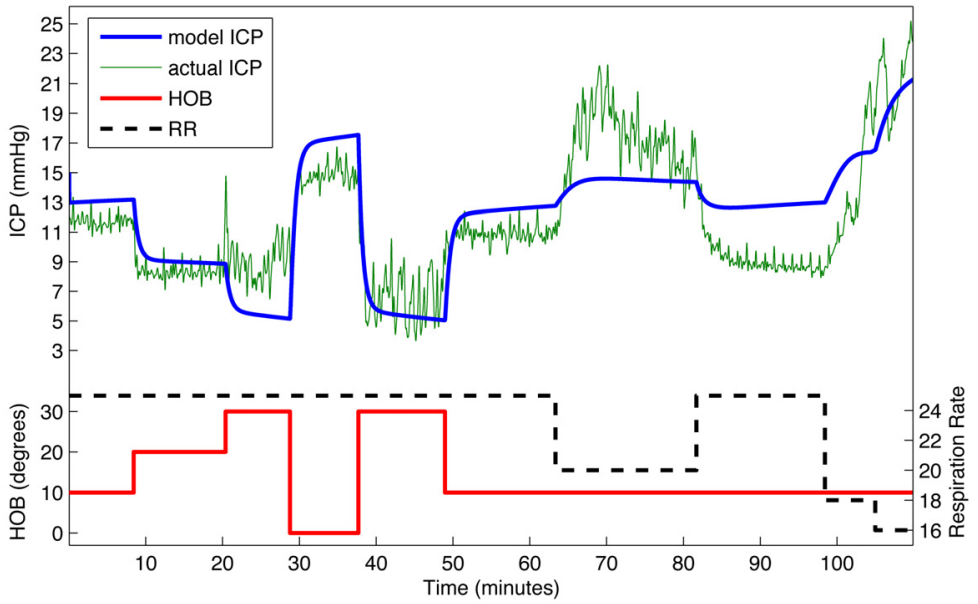

Figure 5: Patient 2, Session 7. A series of changes to HOB elevation and RR.

Finally, although the results presented here demonstrate that the in silico model is capable of being tuned to accurately reflect ICP behavior in specific subjects during specific clinical events, we are concerned that the current optimization process manipulates parameters that may not correlate with any known physiologic process or mechanism. This is only acceptable for a proof of concept model. We expect that future work will lead to a stronger connection between physiology and optimization, while also simplifying the process and bringing us closer to our goal - a virtual patient that responds realistically to different treatment strategies. 


\section{Acknowledgement}

This research was sponsored, in part, by the Thrasher Research Fund.

\section{References}

[1] White, J., Dalton, H., Pediatric Trauma: Post-Injury Care in the Pediatric Intensive Care Unit. Crit Care Med 2002. 30:S478-S488.

[2] Teasdale, G. and Jennett, B., Assessment of coma and impaired consciousness. A practical scale. Lancet 1974. 2(7872):81-4.

[3] Adelson, P., Bratton, S., Carney, N., Chesnut, R., du Coudray, H., Goldstein, B., et al., Guidelines for the acute medical management of severe traumatic brain injury in infants, children and adolescents. Ped Crit Care Med 2003. 4 [3].

[4] Rosner, M., Pathophysiology and management of increased intracranial pressure. In: Neurosurgical Intensive Care, B.T. Andrew (ed), McGrawHill, Inc, 1993. 57-112.

[5] Marmarou, A., Shulman, K., Computer modeling of CSF Pressure/Volume and its relationship to hydrocephalus. Intracranial Pressure 1972. M. Brock, H. Dietz. Berlin. Springer-Verlag. 275-279.

[6] Czosnyka, M., Piechnik, S., Richards, H., Kirkpatrick, P., Smielewski, P., Pickard, J., Contribution of mathematical modeling to the interpretation of bedside tests of cerebrovascular autoregulation. J Neurosurg 1997. 63:721-731.

[7] Ursino, M., Iezzi, M., Stocchetti, N., Intracranial Pressure Dynamics in Patients with Acute Brain Damage: A Critical Analysis with the Aid of a Mathematical Model. IEEE Transactions on Biomedical Engineering 1995. 42:529-540.

[8] Ursino, M., Magosso, E., Role of tissue hypoxia in cerbrovascular regulation: a mathematical modeling study. Ann. of Biomedical Engr. 2001. 29:563-574.

[9] Ursino, M., Lodi, C., A Simple mathematical model of the interaction between intracranial pressure and cerebral hemodynamics. J. Appl. Physiol 1997. 82[4]:1256-1259.

[10] Wakeland, W., McNames, J., Aboy, M., Holleman, D., Goldstein, B., Modeling intracranial fluid flows and volumes during traumatic brain injury to better understand pressure dynamics. Annual Int'l Conf of IEEE Engr in Medicine and Biology-Proc. Cancun, Mexico, pp 402-405, 17-21 Sept. 2003.

[11] Wakeland, W., Goldstein, B., A Computer Model of Intracranial Pressure Dynamics during Traumatic Brain Injury that Explicitly Models Fluid Flows and Volumes. Acta Neurochirurgica Supplemental 2005 (in press).

[12] Wakeland, W., McNames, J., Goldstein, B., Calibrating an intracranial pressure dynamics model with annotated clinical data: a progress report. Annual Int'l Conf. of IEEE Engr. in Medicine and Biology-Proc., San Francisco, California, pp 746-749, 1-5 Sept. 2004. 